\title{
ADAMTS13, Thrombotic Thrombocytopenic Purpura and Pregnancy
}

Agnès Veyradier ${ }^{1,2-5 *}$, Alain Stepanian ${ }^{2,3}$ and Paul Coppo ${ }^{4,5}$

${ }^{1}$ Service d'Hématologie biologique, Hôpital Antoine Béclère, Hôpitaux Universitaires Paris Sud, Assistance Publique-Hôpitaux de Paris, Clamart, France ${ }^{2}$ Inserm U770, Université Paris 11, Le Kremlin Bicêtre, France

${ }^{3}$ Service d'Hématologie biologique, Hôpital Louis Mourier, Hôpitaux Universitaires Paris Nord-Val de Seine, Assistance Publique-Hôpitaux de Paris, Colombes, France ${ }^{4}$ Département d'Hématologie clinique, Hôpital Saint Antoine, Hôpitaux Universitaires de l'Est parisien, Assistance Publique-Hôpitaux de Paris, UPMC (Université Paris 6),

Paris, France

${ }^{5}$ Centre National de Référence des MicroAngiopathies Thrombotiques (CNR-MAT), Paris, France

\section{Abstract}

Thrombotic thrombocytopenic purpura (TTP) is a thrombotic microangiopathy (TMA) which pathophysiology mainly relies on a severe deficiency (either acquired or inherited) of ADAMTS13, the specific von Willebrand factor (VWF) protease. TTP is characterized by a feminine predominance and pregnancy is a precipitating factor for TTP boots. Obstetrical TTP represents at least $20 \%$ of all TTP occurring in child-bearing age women.

In this review, an analyze of the English-language literature from 1955 to 2011 found about 350 cases of obstetrical TTP including about 40 case-reports/-series with well documented ADAMTS13 investigation (32 inherited and 17 acquired TTP with severe ADAMTS13 deficiency). In the 32 patients with inherited TTP, the first pregnancy was systematically associated with a TTP boot, mostly occurring during the third trimester; curative plasma therapy (PT) allowed a good maternal outcome although the fetal outcome was almost systematically bad. In the 17 patients with acquired TTP, TTP also occurred mostly de novo during the first pregnancy and after 20 weeks gestation; curative PT usually allowed a good maternal outcome and the birth of an alive baby in about 2 cases/3.

The diagnosis of obstetrical TTP is challenging because it mostly occurs in women with no antecedent of TTP and it has no specific clinical/biological symptoms except a severely deficient ADAMTS13. However, because of the severity of the prognosis in the absence of urgent treatment, any thrombocytopenia +/- hemolytic anemia in a pregnant woman with no alternative diagnosis to TMA should be considered as TTP.

The management of an obstetrical TTP boot consists in a blood collection for ADAMTS13 investigation followed by an emergency first-line treatment with PT yielding a maternal response rate of about $80 \%$ although the global stillbirth rate is likely to be close to $50 \%$.

The follow-up of women who recovered from an obstetrical TTP boot should include a complete ADAMTS13 investigation to distinguish between the inherited and the acquired form of TTP, in order to both estimate the risk for relapse and optimize prophylaxis indication during subsequent pregnancies. The relapse rate appears to be $100 \%$ in inherited TTP and about $20 \%$ in acquired TTP. Early prophylactic PT is thus indicated systematically in inherited TTP as it is clearly beneficial for both the mother and the fetus outcomes. In contrast, the optimal management is still debated in subsequent pregnancies of women with acquired TTP whose clinical and biological monitoring should be very careful.

TTP is a very rare complication of pregnancy (about 1/100,000 pregnancies) but it is a life-threatening disease for both the mother and the fetus. Many advances have been performed in the last 10 years in terms of diagnosis and treatment. However, clear guidelines are still needed to optimize the management of subsequent pregnancies, which may be significantly different as a function of the pathophysiology for ADAMTS13 deficiency.

\section{Introduction}

Thrombotic Thrombocytopenic purpura (TTP) is a thrombotic microangiopathy (TMA) defined by a microangiopathic hemolytic anemia and thrombocytopenia without an alternative cause [1,2]. Clinical manifestations of multivisceral ischemia (neurologic symptoms, renal and cardiac involvement...) are later events. In about half cases, TTP occurs in patients with previously or concomitantly diagnosed other clinical conditions (pregnancy, infections, autoimmune diseases, drugs, hematopoietic stem cell transplantation, cancer, malignant hypertension...) although in the other half of cases, TTP is apparently idiopathic $[1,2]$. TTP, especially idiopathic forms, is strongly associated with a severe functional deficiency (activity $<10 \%$ ) of ADAMTS13 (A Disintegrin And Metalloprotease with ThromboSpondin type 1 repeats, member 13), the specific metalloprotease that cleaves ultralarge (UL) multimers of von Willebrand factor (VWF), the most hemostatically active species of VWF [3]. Thus, the pathophysiology for about $75 \%$ of TTP is explained by the accumulation of platelet-hyperadhesive ULVWF multimers leading to the spontaneous formation of microthrombi within the microcirculation $[4,5]$. ADAMTS13 severe deficiency may be due either to auto-antibodies to ADAMTS13 (acquired autoimmune TTP) or to recessively inherited bi-allelic mutations of ADAMTS13 gene (hereditary TTP also named Upshaw-Schulman syndrome (USS) [6]. TTP is a rare disease as its prevalence is $4-10$ cases/million people/year. In a large majority of cases, TTP is an adult-onset disease characterized by both a feminine predominance $(2-3 \mathrm{~F} / 1 \mathrm{M})$, a peak between 30 and

*Corresponding author: PrAgnès Veyradier, MD, PhD, Service d'Hématologie biologique Hôpital Antoine Béclère, 157 rue de la Porte-de-Trivaux, 92140 Clamart France, Tel : +33145374305 ; Fax : +331 453740 95; E-mail : agnes.veyradier@abc.aphp.fr

Received December 23, 2011; Accepted February 14, 2012; Published February 19,2012

Citation: Veyradier A, Stepanian A, Coppo P (2012) ADAMTS13, Thrombotic Thrombocytopenic Purpura and Pregnancy. Hereditary Genetics S1:002. doi:10.4172/2161-1041.S1-002

Copyright: (c 2012 Veyradier A, et al. This is an open-access article distributed under the terms of the Creative Commons Attribution License, which permits unrestricted use, distribution, and reproduction in any medium, provided the original author and source are credited. 
40 years-old and more than $95 \%$ of acquired autoimmune forms. TTP usually occurs by recurrent boots separated by remission phases. The global mortality rate is estimated at $20 \%$ in spite of both plasma therapy (PT) which remains the reference treatment, and more recently, the addition of immunomodulating agents like Rituximab $[1,2]$.

TTP occurring in an obstetrical context (pregnancy and postpartum) is an exciting entity as it presents several specificities and challenges. From 1955 to 2011, about 350 cases of obstetrical TTP or TTP/HUS have been counted in the English-language literature including 3 main general reviews [7-9]. These cases were very well clinically documented and most of them were in favor of an acquired form of TMA. However, the large majority of these cases were not investigated for ADAMTS13. In the last 10 years indeed, about 40 obstetrical TTP cases associated with a well documented severe ADAMTS13 deficiency (either acquired or hereditary) have been published in case-reports and case-series studies. This review will describe the miscellaneous aspects of pregnancy-associated TTP including epidemiology, pathophysiology, diagnosis, management, outcome and follow-up. A focus on TTP related to a severe ADAMTS13 deficiency including specificities related to the acquired or inherited feature of TTP will be performed.

\section{Epidemiology and pathophysiology}

On an obstetrical point of view, TTP is reported to complicate $1 / 25000$ to $1 / 100000$ pregnancies worldwide $[7,8,9]$. This very broad range may be explained by the heterogeneity of cases reported in the literature consisting either in "TTP/HUS" patients or miscellaneous "TTP" patients whose ADAMTS13 was not systematically investigated. Consequently, one can speculate that TTP associated with a well characterized severe ADAMTS 13 functional deficiency may not occur in more than $(1 / 100000)$ pregnancies and thus remains a very rare although life-threatening complication of pregnancy.

On a point of view focused on TMA, North American, European and Japanese registries report that pregnancy-associated TTP represents 10 to $30 \%$ of all adult TTP $[8,10-15]$. As expected, the rates higher than $20 \%$ are found in studies considering pregnancy-associated TTP only among child-bearing age women and they are certainly the most relevant. Interestingly, in a large majority of women with a pregnancyassociated TTP, the current TTP boot is the first manifestation of the disease [9].

The strong association between TTP and pregnancy may be explained by several mechanisms. First, it may be a consequence of the feminine predominance of TTP (being itself at least partially linked to the feminine predominance classically observed in any disease with an autoimmune background); furthermore, most women with TTP (between 60 and 70\%) are child-bearing age women [1,2]. Second, pregnancy is associated with physiological coagulation changes predisposing to hypercoagulability and particularly to a dysbalance of the VWF/ADAMTS13 system: indeed, during the course of pregnancy, VWF levels in plasma increase progressively to reach levels $2.5-3$ fold higher levels at term (with peak values occurring immediately following delivery) while ADAMTS13 decreases progressively (ADAMTS13 activity decreases of about $30 \%$ at term when compared to baseline levels before pregnancy) (Supplementary Table 1) [16-23]. The physiological decrease of ADAMTS13 during pregnancy may be due either to a consumption mechanism by its VWF substrate [16], or to a direct effect of hormonal substances on ADAMTS13 metabolism [24]. However, the physiological decrease of ADAMTS13 during normal pregnancy remains well above $10 \%$ (only ADAMTS13 values lower than the latter threshold are strongly associated with TTP $[1,2,3]$. In contrast, in case of severe ADAMTS13 functional deficiency, the physiological significant increase of VWF during the second trimester of gestation may act as a crucial triggering factor for a TTP boot. Indeed, TTP essentially occurs from the second third of pregnancy (second and third trimesters) and sometimes in post-partum [7], while it remains unusual during the first trimester of gestation $[25,26]$.

\section{Specific features of pregnancy-associated TTP at presentation as a function of ADAMTS13 deficiency etiology}

Pregnancy-associated TTP in women with hereditary TTP (USS): Data collected from publications between 1976 and 2011 found 32 patients with pregnancy-associated hereditary TTP (USS). In 8 patients, ADAMTS13 was not investigated but both clinical features and familial occurrence supported the diagnosis [7]. In 24 patients [2736], ADAMTS13 was documented (ADAMTS13 activity lower than $10 \%$ in the absence of auto-antibodies) including 15 cases in whom ADAMTS13 genotype could also be characterized (Supplementary Table 2) $[30,32,34,36]$. Interestingly, all 6 cases reported from the Western World [30,32,36] carried the R1060W mutation (either heterozygous or homozygous), reported to be a marker for adult TTP [32], and they were indeed associated with an adult-onset USS initially recognized with the first pregnancy (Supplementary Table 2). In contrast, the largest series of 9 pregnancy-associated USS reported in Japan by Fujimura et al [34], showed miscellaneous mutations not including the R1060W ADAMTS13 variant: interestingly, 6 of these 9 women had episodes of severe to mild thrombocytopenia during childhood that had been incorrectly diagnosed as ITP while 3 patients had an adult-onset TTP initially recognized with pregnancy. Whatever may be the age of TTP onset, the first pregnancy was complicated by a TTP boot in all Japanese patients (Supplementary Table 2).

About 50 pregnancies could be documented from these 32 patients and showed that in almost $75 \%$ of cases, TTP occurred during the $3^{\text {rd }}$ trimester of pregnancy (Supplementary Table 2). Considering the first pregnancy, thanks to curative PT performed in all cases from the 1990's, the maternal outcome consisted in remission without major sequellae; in contrast, the fetal outcome was dramatic consisting in intra-utero fetal death (IUFD) (miscarriage, stillbirth) or infant early death. In very rare cases however, the TTP boot occurring during the first pregnancy led to a curative PT maintained until delivery and allowing the birth of an alive baby (Supplementary Table 2) $[27,31,34,35]$.

\section{Pregnancy-associated TTP in women with acquired autoimmune TTP}

Analysis of papers published from 2002 to 2011 shows 17 detailed case-reports $[8,31,37-46]$, of acquired TTP where ADAMTS13 was documented (Supplementary Table 3).

In $12 / 17$ cases (70\%), TTP occurred during the second half of pregnancy (after $20 \mathrm{WG}$ ) including 7 cases during the $3^{\text {rd }}$ trimester and 2 cases during the post-partum. In $14 / 17$ patients (82\%), the TTP boot occurred during the first pregnancy including 11 patients with no antecedent of TTP and 3 patients with either previous TTP boots or autoimmunity $[29,31,40,44]$. In $3 / 17$ patients $(18 \%)$, the TTP boot occurred during the second or the third pregnancy including either patients with antecedent of TTP $[8,41]$ or not [42]. At presentation, in all patients, ADAMTS13 activity was lower than $10 \%$ and associated with detectable auto-antibodies in a large majority of cases $(n=12)$; the acquired feature of TTP was however attested by later normalization of ADAMTS13 activity in most cases.

In most documented cases, PT was systematically performed as 
a curative treatment, sometimes with additional immunomodulators (steroids, cyclosporine, rituximab) allowing a maternal survival. Three patients received platelet infusion and one of them died [42]. The fetal outcome showed 11 alive babies out of 15 documented cases. The good fetal outcome looks correlated to the term of pregnancy where TTP occurs because it is usually associated with late $3^{\text {rd }}$ trimester boots (Supplementary Table 3).

\section{Diagnosis of TTP during pregnancy}

As previously described, either in hereditary or acquired ADAMTS13 severe deficiency, pregnancy-associated TTP occurs mostly in women with no antecedent of TMA. Consequently, at presentation, to reach the diagnosis of TTP is challenging and it should proceed in two main steps: first, the recognition of a TMA among the numerous causes of pregnancy-associated thrombocytopenia [46], and second the differential diagnosis with other pregnancy-associated TMA like preeclampsia (PE), the HELLP (Hemolysis Elevated Liver Enzyme Low Platelet count) syndrome or the hemolytic uremic syndrome (HUS) [47].

The most constant sign of TTP is thrombocytopenia [1,2]. During pregnancy, thrombocytopenia occurs commonly (6 to $10 \%$ of all pregnant women) and may be due to miscellaneous etiologies, some of which being unique to pregnancy (mainly gestational thrombocytopenia, PE, HELLP syndrome) while others may also occur in a non obstetrical context (mainly primary or secondary immune thrombocytopenia, TTP, HUS, disseminated intravascular coagulation (DIC) [46].

At presentation, the exclusion of gestational thrombocytopenia, secondary immune thrombocytopenia (Evans syndrome) or DIC is usually performed easily thanks to the severity of thrombocytopenia (usually lower than $50 \mathrm{G} / \mathrm{L}$ ), negative direct Coomb's test and normal coagulation profile, respectively. Primary immune thrombocytopenia may also be excluded because of the presence of mechanical hemolytic anemia (shistocytes on peripheral blood smear) specifically in TTP. In contrast, other TMA syndromes like PE, HELLP and HUS may be very challenging to distinguish from TTP because they may share many overlapping features like the microangiopathic anemia, the multivisceral ischemia leading to end stage organ failure (neurologic, renal, cardiac, digestive involvement) as well as hypertension $[7,9,33]$. Also, the term of gestation may not be informative as all these TMA syndromes may occur during the second half of pregnancy. Thus, using clinical symptoms and standard biology, a definitive diagnosis of TTP in a pregnant patient with such manifestation may be impossible to achieve prospectively at presentation.

In contrast, retrospective analysis of pregnancy-associated TMA syndromes shows that several features may distinguish TTP from other TMA. First, unlike PE and the HELLP syndrome, termination of pregnancy (fetal extraction) does not induce remission of TTP/ HUS [46,47]. Second, HUS is the only TMA to be more frequent during post-partum [2]. Indeed, puerperium and post-partum TTP are more rare than ante-partum TTP and they usually occur following a late $3^{\text {rd }}$ trimester delivery (about $38 \mathrm{WG}$ ) and a median $4^{\text {th }}$ day postpartum (range 0-42 days) [9]. Third, several studies led in women with pregnancy-associated TMA showed that a severe ADAMTS13 deficiency (activity $<10 \%$ ) was specific for TTP: indeed, only normal or partially deficient ADAMTS13 levels (usually $>20 \%$ ) have been described in either PE, HELLP syndrome or HUS (Supplementary Table 4) [17,19,21-23]. ADAMTS13 activity measurement remains however unavailable in emergency as a routine assay and it may not be useful to argue for a TTP diagnosis at presentation in emergency.
Consequently, as neither clinical features nor biological parameters are able to specifically identify TTP in emergency and because of the severity of the prognosis in the absence of urgent appropriate treatment (PT), any pregnant patient with thrombocytopenia and mechanical hemolytic anemia with no other alternative diagnosis than TMA should be considered to have TTP and treated accordingly $[7,9,46,47]$.

In more rare cases, pregnancy-associated TTP occur in women with TTP antecedents $[8,34,41,42]$. In these women, the diagnosis of TTP at presentation is less challenging as pregnancy is established to be a precipitating factor for TTP $[1,2]$.

\section{Management and outcome of a TTP boot during pregnancy}

Laboratory investigation: Before to initiate any treatment, especially in women whose TTP came de novo during pregnancy, venous blood should be collected to measure ADAMTS13-related parameters (ADAMTS13 activity and anti-ADAMTS13 IgG): indeed, even if not available in emergency in most hospitals, ADAMTS13 investigation is useful as it will allow to both confirm the diagnosis of ADAMTS13 severe deficiency and, in most cases, identify anti-ADAMTS13 autoantibodies supporting the diagnosis of acquired autoimmune TTP. In some cases however, anti-ADAMTS13 auto-antibodies may not be detectable during the TTP boot [2]. Thus, only further ADAMTS13 testing in remission including ADAMTS13 activity, anti-ADAMTS13 auto-antibodies and if necessary, ADAMTS13 gene sequencing, will allow the differential diagnosis between acquired and inherited TTP (see infra).

Maternal therapeutic management and outcome: The UK guidelines for the management of TTP in pregnancy (curative treatment) recommend to treat with plasma exchange as non pregnant patients (Grade C, level IV) [48], this treatment yielding a response rate of about $80 \%$ similar to that observed globally in TTP [46]. The maternal prognosis of patients with post-partum-onset TTP is similar to that of patients with ante-partum-onset TTP (average mortality rate of 20\%) [9]. In contrast, the combination of TTP and PE/HELLP syndrome significantly increases maternal mortality (44\%) [9]. The immune background of most TTP also provides a rationale for the use of corticosteroids: however, their benefit in TTP has not been determined through randomized studies and they are associated with an increased risk of complication in pregnant individuals [46]. In contrast to PE or the HELLP syndrome, delivery is recommended only for those women who do not respond to plasma exchange (Grade C, level IV) [48]. Also, in those women who do not correctly respond to this first line treatment, the indication of rituximab, a chimeric monoclonal antibody directed against B-cell surface antigen CD20, may be debated. Rituximab is indicated for some hematologic malignancies and rheumatoid arthritis but it has also been successfully used in other autoimmune diseases like lupus erythematosous, idiopathic thrombocytopenic purpura or TTP [49]. However, a review of the literature focused on the use of rituximab in 231 pregnancies shows that it is able to cross the placenta and may induce hematologic abnormalities or malformation in the neonates [50]. The use of rituximab in a pregnancy-associated TTP has been reported in only one patient [31], the indication was a refractory TTP of the $3^{\text {rd }}$ trimester of pregnancy. No maternal or fetal toxicity were observed.

Fetal outcome: Globally, the stillbirth rate in pregnancyassociated TTP is reported to be about $40 \%$ mainly due to IUFD and/ or spontaneous abortions and prematurity [9]. In pregnant women with initial TTP, the result of the fetus ultrasonographic evaluation appears dependent on the pregnancy term where the TTP boot occurs: at presentation, most early-term TTP ( $1^{\text {st }}$ and $2^{\text {nd }}$ trimesters $)$ 
are associated with an IUFD [7,9] (Supplementary Tables 2 and 3) although the $3^{\text {rd }}$ trimester perinatal loss is reported to be $17 \%$ since the initiation of PT in 1996 [9]. This 17\% rate remains global and may be underestimated because calculated from patients with TMA diagnosed as TTP on clinical features but not investigated for ADAMTS13. In rare cases however, $1^{\text {st }}$ and $2^{\text {nd }}$ trimester-TTP were not associated with IUFD but led to delivery of alive babies from 32 WG thanks to continuous PT until post-partum $[31,34,35,43]$. This observation makes likely the benefit of maternal PT also for the fetus. Indeed, by replacement of ADAMTS13 in both the maternal and fetal circulation, PT may reduce the placental micro-occlusion arterial process observed in rare TTP cases where a placental anatomopathology investigation was performed [33-35,51]. This placental ischemia may indeed be a major pathophysiological mechanism for intrauterine fetal growth retardation and death observed in obstetrical TTP [35]. Interestingly, no case of fetal thrombocytopenia or haemolytic anemia has been described in maternal TTP although the molecular weight of anti-ADAMTS13 IgG allow them to cross the placental barrier. In only one case of maternal autoimmune acquired TTP, blood analysis from the neonate showed a reduced ADAMTS13 activity of $15 \%$ together with a detectable anti-ADAMTS13 IgG [43]. However, the neonate's platelet count and hemoglobin level were normal.

\section{Follow up of Women who Recovered from a Pregnancy- Associated TTP}

\section{ADAMTS13 investigation to distinguish between acquired and inherited TTP}

After recovery from a pregnancy-induced TTP with severe ADAMTS13 deficiency, a first crucial step of the follow-up should focus on making a differential diagnosis between an acquired and an inherited form of the disease. Indeed, to elucidate the cause of ADAMTS13 deficiency (either genetic or autoimmune) is essential for the patient as it conditions the outcome of the disease in terms of risk of relapse (especially during the subsequent pregnancies, see infra) and further both curative and prophylactic treatments (resort to immunomodulators only in case of acquired TTP for example). In addition, in case of USS, a familial inquiry should also be performed (especially in asymptomatic never-child bearing sisters of pregnancyonset USS patients).

In that regard, combined analysis of ADAMTS13-related parameters during the TTP boot and in remission is necessary to identify the cause of ADAMTS13 severe deficiency. In some cases, the diagnosis of acquired autoimmune TTP may be highly suspected as soon as the TTP boot if ADAMTS13 severe deficiency is associated with anti-ADAMTS13 IgG. In contrast, if anti-ADAMTS13 IgG are not detectable during the TTP boot, the differential diagnosis between acquired and inherited TTP cannot be performed. Thus, ADAMTS13 investigation in remission may be helpful: 1/ if ADAMTS13 activity has become detectable, the diagnosis of acquired TTP may be established; 2/ if ADAMTS13 activity is still undetectable, ADAMTS13 gene sequencing should be performed to confirm the suspicion of USS.

\section{Risk for Relapse and Prophylaxis during Subsequent Pregnancies}

Thus, a second crucial concern for the patient is the risk for relapse, especially during subsequent pregnancies and consequently, the methods for an appropriate prophylaxis. Data from the literature suggest that the risk for relapse and the therapeutic management during subsequent pregnancies are quite different as a function of the cause of ADAMTS13 deficiency.

In case of inherited TTP (USS), the risk for relapse is 100\% in the subsequent pregnancy in the absence of prophylaxis [8,34]. Analysis of the literature (Supplementary Table 2) clearly shows the benefit of prophylactic PT (either plasma exchange or, rather, plasma infusion) which should now be performed systematically in pregnant women with known USS [31,33-35,36]. In those patients, prophylactic PT should be initiated as soon as the end of the $1^{\text {st }}$ trimester (about $10 \mathrm{WG}$ or maybe before) until early post-partum to prevent TTP boots as well as severe fetal complications due to placental microinfarctions. In some cases, PT was empirically associated with steroids, aspirin [31,33-35],or LMWH $[31,36]$. Additional studies would be however useful to better clarify the efficacy of such medications in this context. In exceptional cases however, uneventful pregnancies and deliveries were possible with no prophylactic PT but with aspirin [33,34]. Interestingly, no case of allo-antibodies to ADAMTS13 has ever been described in patients with USS in spite of regular prophylactic PT. In addition, pregnant women with USS should have folic acid supplementation because of latent continuous hemolysis. They should also be immunized against hepatitis B [9].

In case of acquired TTP, the accurate risk of recurrence in subsequent pregnancies remains unknown because of limited reports $[1,7]$. Globally, this risk was reported to be about $20 \%$ when considering the Oklahoma registry confronted to a review of the literature [8]. However, this risk is probably dependent on both ADAMTS13 activity just before pregnancy initiation and the time course of ADAMTS13 activity until delivery. In women who clinically recovered from a TTP but who are diagnosed with a severely deficient ADAMTS13 activity at the onset of pregnancy, regular plasma exchange (at least fortnightly) and serial monitoring of ADAMTS13 activity were reported to have a benefit for both the maternal and the baby outcome in a few patients [31]. However, the experience of systematic use of prophylactic PT during the whole pregnancy in women with a previously diagnosed acquired TTP (either pregnancy-induced or not) remains extremely limited. Forthcoming studies should establish whether therapeutic schedules including immunomodulatory drugs similar to the treatment for lupus could be safely used in this context. In contrast, during the puerperium and a few days during the post-partum, prophylactic PT is usually performed to prevent a TTP boot because delivery is highly suspected to be a trigger for TTP relapse $[1,2,7]$. Also, there is only anecdotal evidence to suggest that the use of anti-platelets agents or steroids may prevent TTP relapse during subsequent pregnancies in women with an antecedent of acquired TTP [48].

\section{Conclusion}

This review of the literature clearly shows a better awareness of the "TTP option" among the miscellaneous TMA associated with pregnancy for the last 10 years. The curative management of a de novo obstetrical TTP is well established and allows a good maternal outcome in a large majority of cases although the stillbirth rate remains high. Concerning the risk for relapse in subsequent pregnancies, the analysis of the literature emphasizes that inherited and acquired TTP may constitute two distinct entities. Inherited TTP (USS) is associated with a very high risk for relapse and severe fetal complications, and evidencebase medicine clearly supports the benefit of an early prophylactic PT for both the mother and the fetus. In contrast, there is still no clear guideline for prophylaxis during pregnancy in women with an antecedent of acquired TTP probably because: 1 / the risk for relapse is not predictable and not clearly evaluated yet (in that regard, the interest 
of ADAMTS13 time course as a prognosis marker has to be studied); $2 /$ the benefit of PT may be balanced to the iatrogenic risk of re-boost of the anti-ADAMTS13 autoantibody; 3/ the alternative therapeutic approaches i.e. steroids, aspirin, LMWH have been only poorly and empirically used. Further studies supported by TTP registries are strongly needed to answer these crucial questions about obstetrical TTP, in order to better define appropriate recommendations validated by multidisciplinary teams involving intensive care unit physicians, hematologists, obstetricians and pediatricians.

\section{References}

1. George JN (2006) Thrombotic thrombocytopenic purpura. N Engl J Med 354 1927-1935.

2. Zheng XL, Sadler JE (2008) Pathogenesis of thrombotic microangiopathies. Annu Rev Pathol 3: 249-277.

3. Tsai HM (2010) Pathophysiology of thrombotic thrombocytopenic purpura. Int $J$ Hematol 91: 1-19.

4. Furlan M, Robles R, Galbusera M, Remuzzi G, Kyrle PA, et al. (1998) von Willebrand factor-cleaving protease in thrombotic thrombocytopenic purpura and the hemolytic-uremic syndrome. N Engl J Med 339: 1578-1584.

5. Tsai HM, Lian EC (1998) Antibodies to von Willebrand factor cleaving protease in acute thrombotic thrombocytopenic purpura. N Engl J Med 339: 1585-1594.

6. Levy GG, Nichols WC, Lian EC, Foroud T, Mc Clintick JN, et al. (2001) Mutations in a member of the ADAMTS gene family cause thrombotic thrombocytopenic purpura. Nature 413: 488-494.

7. George JN (2003) The association of pregnancy with thrombotic thrombocytopenic purpura-hemolytic uremic syndrome. Curr Opin Hematol 10: 339-344.

8. Vesely SK, Li X, McMinn JR, Terrell DR, George JN (2004) Pregnancy outcomes after recovery from thrombotic thrombocytopenic purpura-haemolytic uremic syndrome. Transfusion 44: 1149-1158.

9. Martin JN Jr, Bailey AP, Rehberg JF, Owens MT, Keiser SD, et al. (2008) Thrombotic thrombocytopenic purpura in 166 pregnancies: 1955-2006. Am J Obstet Gynecol 199: 98-104.

10. Rock GA, Shumak KH, Buskard NA, Blanchette VS, Kelton JG, et al. (1991) Comparison of plasma exchange with plasma infusion in the treatment of thrombotic thrombocytopenic purpura. Canadian Apheresis Study Group N Engl J Med 325: 393-397.

11. Noris M, Remuzzi RG (2010) Genetics and genetic testing in haemolytic uremic syndrome/thrombotic thrombocytopenic purpura. Semin Nephrol 30: 395-408.

12. Lotta LA, Mariani M, Consonni D, Mancini I, Palla R, et al. (2010) Different clinical severity of first episodes and reccurrences of thrombotic thrombocytopenic purpura. Br J Haematol 151: 488-494.

13. George JN, Kremer Hovinga J, Terell DR, Vesely SK, Lammle B (2008) The Oklahoma Thrombotic thrombocytopenic purpura-hemolytic uremic syndrome registry: the swiss connection. Eur J Haematol 80: 277-286.

14. Scully M, Yarranton H, Liesner R, Cavenagh G, Hunt B, et al. (2008) Regional UK TTP registry: correlation with laboratory ADAMTS13 analysis and clinical features. Br J Haematol 142: 819-826.

15. Fujimura Y, Matsumoto M (2010) Registry of 919 patients with thrombotic microangiopathies across Japan: database of Nara medical university during 19982008. Intern Med 49: 7-15.

16. Mannucci PM, Canciani MT, Forza I, Lussana F, Lattuada A, et al. (2001) Changes in health and disease of the metalloprotease that cleaves von Willebrand factor. Blood 98: 2730-2735

17. Lattuada A, Rossi E, Calzarossa C, Candolfi R, Mannucci PM (2003) Mild to moderate reduction of a von Willebrand factor cleaving protease (ADAMTS-13) in pregnant women with HELLP microangiopathic syndrome. Haematologica 88 1029-1034

18. Sánchez-Luceros A, Farías CE, Amaral MM, Kempfer AC, Votta R, et al. (2004) von Willebrand factor-cleaving protease (ADAMTS13) activity in normal non-pregnan women, pregnant and post-delivery women. Thromb Haemost 92: 1320-1326.

19. Hulstein JJ, van Runnard Heimel PJ, Franx A, Lenting PJ, Bruinse HW, et al (2006) Acute activation of the endothelium results in increased levels of active von Willebrand factor in hemolysis, elevated liver enzymes and low platelets (HELLP) syndrome. J Thromb Haemost 4: 2569-2575.
20. Feys HB, Canciani MT, Peyvandi F, Deckmyn H, Vanhoorelbeke K, et al. (2007) ADAMTS13 activity to antigen ratio in physiological and pathological conditions associated with an increased risk of thrombosis. Br J Haematol 138: 534-540.

21. Molvarec A, Rigo Jr, Boze T, Derzsy Z, Cervenak L, et al. (2009) Increased plasma von Willebrand factor antigen levels but normal von Willebrand factor cleaving protease (ADAMTS13) activity in preeclampsia. Thromb Haemost 101: 305-311.

22. Stepanian A, Cohen-Moatti M, Sanglier T, Legendre P, Ameziane N, et al. (2011) ECLAXIR study group Von Willebrand factor and ADAMTS13: a candidate couple for the preeclampsia pathophysiology. Arterioscl Thromb Vasc Biol 31: 1703-1709.

23. Alpoim PN, Gomes KB, Godoi LC, Rios DR, Carvalho MG, et al. (2011)ADAMTS13 FVIII von Willebrand factor, ABO blood group assessment in preeclampsia. Clin Chim Acta 412: 2162-2166.

24. Powazniak Y, Kempfer AC, Pereyra JC, Palomino JP, Lazzari MA (2011) VWF and ADAMTS13 behaviour in estradiol treated HUVEC. Eur J Haematol 86: 140-147.

25. Sharma A, Radotra B, Wanchu A, Malhotra P, Singh S, et al. (2008) Thrombotic thrombocytopenic purpura in a patient with very early pregnancy: clinical presentation and autopsy findings. Blood Coagul Fibrinolysis 19: 727-730.

26. Trisolini SM, Capria S, Gozzer M, Pupella S, Foa R, et al. (2009) Thrombotic thrombocytopenic purpura in the first trimester and successful pregnancy. Ann Hematol 88: 287-289.

27. Furlan M, Robles R, Solenthaler M, Wassmer M, Sandoz P, et al. (1997) Deficien activity of von Willebrand factor-cleaving protease in chronic relapsing thrombotic thrombocytopenic purpura. Blood 89: 3097-3103.

28. Furlan M, Lämmle B (2001) Aetiology and pathogenesis of thrombotic thrombocytopenic purpura and haemolytic uraemic syndrome: the role of von Willebrand factor-cleaving protease. Best Pract Res Clin Haematol 14: 437-454

29. Sivakumaran M, Roland J (2002) Prophylactic treatment with fresh-frozen plasma in chronic thrombotic thrombocytopenic purpura. Br J Haematol 117: 480-483.

30. Donadelli R, Banterla F, Galbuserra M, Capoferri C, Bucchioni S, et al. (2006) International Registry of Reccurrent and Familial HUS/TTP. In-vitro and invivo consequences of mutations in the von Willebrand factor cleaving protease ADAMTS13 in thrombotic thrombocytopenic purpura. Thromb Haemost 96: 454 464

31. Scully M, Starke R, Lee R, Mackie I, Machin S, et al. (2006) Successful management of pregnancy in women with a history of thrombotic thrombocytopaenic purpura. Blood Coagul Fibrinolysis 17: 459-463.

32. Camillieri RS, Cohen H, Mackie IJ, Scully M, Starke RD, et al. (2008) Prevalence of the ADAMTS-13 missense mutation R1060W in late onset adult thrombotic thrombocytopenic purpura. J Thromb Haemost 6: 331-338.

33. Kato R, Shinohara A, Sato J (2009) ADAMTS13 deficiency, an important cause of thrombocytopenia during pregnancy. Int J Obstet Anesth 18: 73-77.

34. Fujimura Y, Matsumoto M, Kokame K, Isonishi A, Soejima K, et al. (2009) Pregnancy-induced thrombocytopenia and TTP, and the risk of fetal death, in Upshaw-Schulman syndrome: a series of 15 pregnancies in 9 genotyped patients Br J Haematol 144: 742-754.

35. Meti S, Paneesha S, Patni S (2010) Successful pregnancy in a case of congenital thrombotic thrombocytopenic purpura. J Obstet Gynaecol 30: 519-521.

36. Richter J, Strandberg K, Lindblom A, Strevens H, Karpman D, et al. (2011) Successful management of a planned pregnancy in severe congenital thrombotic thrombocytopaenic purpura: the Upshaw-Schulman syndrome. Transfus Med 21: 211-213.

37. He Y, Chen Y, Zhao Y, Zhang Y, Yang W (2010) Clinical study on five cases of thrombotic thrombocypenic purpura complicating pregnancy. Aust N Z J Obstet Gynaecol 50: 519-522.

38. Raman R, Yang S, Wu HM, Cataland SR (2011) ADAMTS13 activity and the risk of thrombotic thrombocytopenic purpura relapse in pregnancy. $\mathrm{Br} \mathrm{J}$ Haematol 13652141.

39. Cosmai EM, Puzis L, Tsai HM, Lian EC (2002) Thrombocytopenic purpura and cardiomyopathy in pregnancy reversed by combined plasma exchange and infusion. Eur J Haematol 68: 239-242.

40. Obeidat B, MacDougall J, Harding K (2002) Plasma exchange in a woman with thrombotic thrombocytopenic purpura or severe pre-eclampsia. BJOG 109: 961 962.

41. Ducloy-Bouthors AS, Caron C, Subtil D, Provot F, Tournoys A, et al. (2003) Thrombotic thrombocytopenic purpura: medical and biological monitoring of six 
Citation: Veyradier A, Stepanian A, Coppo P (2012) ADAMTS13, Thrombotic Thrombocytopenic Purpura and Pregnancy. Hereditary Genetics S1:002. doi:10.4172/2161-1041.S1-002

pregnancies. Eur J Obstet Gynecol Reprod Biol 111: 146-152.

42. Rehberg JF, Briery CM, Hudson WT, Bofill JA, Martin JN Jr (2006) Thrombotic thrombocytopenic purpura masquerading as hemolysis, elevated liver enzymes, low platelets (HELLP) syndrome in late pregnancy. Obstet Gynecol 108: 817-820.

43. Gerth J, Schleussner E, Kentouche K, Busch M, Seifert M, et al. (2009) Pregnancyassociated thrombotic thrombocytopenic purpura. Thromb Haemost 101: 248-251.

44. Watanabe R, Shirai T, Tajima Y, Ohguchi H, Onishi Y, et al. (2010) Pregnancyassociated thrombotic thrombocytopenic purpura with anti-centromere antibodypositive Raynaud's Syndrome. Intern Med 49: 1229-1232.

45. Kovacs EM, MolvarecA, Rigo Jr, Szabo A (2011) Bilateral serous retinal detachment as a complication of acquired peripartum thrombotic thrombocytopenic purpura. $J$ Obstet Gynaecol Res 37: 1506-1509.

46. McCrae KR (2010) Thrombocytopenia in pregnancy. Educ Program Blood Hematology 397-402.
47. Stella CL, Dacus J, Guzman E, Dhillon P, Coppage K, et al. (2009) The diagnosis dilemma of thrombotic thrombocytopenic purpura/haemolytic uremic syndrome in the obstetric triage and emergency department: lessons from 4 tertiary hospitals. Am J Obstet Gynecol 200: 381.e1-381.e6.

48. Allford SL, Hunt BJ, Rose P, Machin SJ (2003) Guidelines on the diagnosis and management of thrombotic microangiopathic haemolytic anemias. $\mathrm{Br} \mathrm{J}$ Haematol 120: 556-573.

49. Barcellini W, Zanella A (2011) Rituximab therapy for autoimmune haematological diseases. Eur J Intern Med 22: 220-229.

50. Chakravarty EF, Murray ER, Kelman A, Framer P (2011) Pregnancy outcomes after maternal exposure to rituximab. Blood 117: 1499-1506.

51. Jamshed S, Kouides P, Sham R, Cramer S (2007) Pathology of thrombotic thrombocytopenic purpura in the placenta, with emphasis on the snowman sign Pediatr Dev Pathol 10: 455-462.
This article was originally published in a special issue, Hereditary Disorders handled by Editor(s). Dr. X. Long Zheng, University of Pennsylvania, USA 